\title{
Manipulation of Positron Orbits in a Dipole Magnetic Field with Fluctuating Electric Fields
}

\author{
H. Saitoh ${ }^{1,5}$, J. Horn-Stanja ${ }^{1}$, S. Niß1 ${ }^{1}$, E. V. Stenson ${ }^{1}$, U. Hergenhahn ${ }^{1,6}$, T. Sunn \\ Pedersen $^{1,7}$, M. Singer ${ }^{2}$, M. Dickmann ${ }^{2}$, C. Hugenschmidt ${ }^{2}$, M. R. Stoneking ${ }^{3}$, J. \\ R. Danielson ${ }^{4}$ and C. M. Surko ${ }^{4}$ \\ ${ }^{1}$ Max Planck Institute for Plasma Physics, Greifswald and Garching, Germany \\ ${ }^{2}$ Technische Universität München, Garching, Germany \\ ${ }^{3}$ Department of Physics, Lawrence University, Appleton, USA \\ ${ }^{4}$ University of California, San Diego, USA \\ ${ }^{5}$ The University of Tokyo, Kashiwa, Japan \\ ${ }^{6}$ Leibniz Institute of Surface Modification, Leipzig, Germany \\ ${ }^{7}$ Ernst Moritz Arndt Universität, Greifswald, Germany
}

\begin{abstract}
We report the manipulation of positron orbits in a toroidal dipole magnetic field configuration realized with electric fields generated by segmented electrodes. When the toroidal circulation motion of positrons in the dipole field is coupled with timevarying electric fields generated by azimuthally segmented outer electrodes, positrons undergo oscillations of their radial positions. This enables quick manipulation of the spatial profiles of positrons in a dipole field trap by choosing appropriate frequency, amplitude, phase, and gating time of the electric fields. According to numerical orbit analysis, we applied these electric fields to positrons injected from the NEPOMUC slow positron facility into a prototype dipole field trap experiment with a permanent magnet. Measurements with annihilation $\gamma$-rays clearly demonstrated the efficient compression effects of positrons into the strong magnetic field region of the dipole field configuration. This positron manipulation technique can be used as one of essential tools for future experiments on the formation of electron-positron plasmas.
\end{abstract}

\section{INTRODUCTION}

In order to experimentally investigate matter-antimatter pair-plasmas $[1,2,3,4,5]$, we aim to create magnetically confined electron-positron plasmas [6, 7] by using the NEPOMUC (NEutron induced POsitron source MUniCh) slow positron facility [8]. Use of toroidal magnetic field configurations is a key to realize simultaneous confinement of electrons and positrons as plasmas. A toroidal dipole magnetic field configuration is suitable for stable confinement of plasmas at any no-neutrality [9] including pair-plasmas. In the dipole field configuration, excellent confinement properties have been demonstrated for both single-component non-neutral plasmas [10] and fusion-oriented hightemperature plasmas [11,12]. In order to trap high density positrons in the dipole field trap, a large number of positrons must be transported into the confinement region of the trap with high efficiency. For this purpose, we plan to accumulate positron beam from NEPOMUC in a buffer gas based linear trap [13], and transport them into a superconducting (SC) levitated dipole field trap. Prior to the construction of the accumulator and SC levitated dipole devices, we are conducting studies to develop injection and manipulation methods of positrons in the dipole magnetic field. In a prototype dipole field trap operated at NEPOMUC, so far we have realized efficient injection of positron beam with a $\mathbf{E} \times \mathbf{B}$ drift scheme [14, 15]. Recent optimization work realized almost loss-less (close to $100 \%$ efficiency) injection. Here development of manipulation techniques of positrons in the UHV (ultra high vacuum) environment, especially the radial compression scheme, is important for the realization of high density states of positrons after injection.

The behavior of charged particles in a dipole magnetic field [16] is characterized by the combination of gyration, bounce along field lines, and toroidal precession motions. Adiabatic invariants related to these three periodic motions are magnetic moment $\mu$ for gyration, longitudinal invariant $J$ for the bounce motion, and canonical angular momentum 
$P_{\theta}$ for the toroidal circulation. In the limit of a strongly magnetized particle, the $P_{\theta}$ conservation is equivalent to the conservation of the magnetic flux function of a dipole field. Because of the conservation of $P_{\theta}$, which is realized by the axisymmetry of the system, the guiding center motions of charged particles in a dipole field are trapped on magnetic surfaces. This is a basis for the excellent confinement properties of the dipole field configuration. On the other hand, in order to generate high density positrons in the dipole field trap, we must break the conservation of $P_{\theta}$ and realize efficient transport of particles toward the strong field region. A common transport mechanism of charged particles in the dipole field is the so-called inward or uphill "diffusion" driven by slow (a time scale comparable to the toroidal precession motion) fluctuations [16]. This is a widely observed robust mechanism for the self-organization processes of charged particles in both laboratory $[17,12,18]$ and planetary [19] magnetospheres.

In experiments to create electron-positron plasmas, however, pulsed positrons from an accumulator are injected into the dipole field trap in a rather short time scale, for example in the order of $\mu \mathrm{s}$. For the shaping of injected positron orbits with avoiding particle loss toward the outer electrodes and the magnet in the initial phase just after injection, it is needed to develop a fast manipulation method of positrons by breaking the $P_{\theta}$ conservation. In this study, we report preliminary results on a new scheme to realize efficient injection and subsequent quick compression of positron orbits by combination of the $\mathbf{E} \times \mathbf{B}$ drift method and application of a temporary gated fluctuating electric fields. In a linear Penning-Malmberg trap, the use of rotating wall (RW) electric fields is an established technique to manipulate single-component plasmas [13] realized through coupling with plasma modes [20] or without strong dependence on the plasma modes in the strong drive regime $[21,22]$. In this study, we applied fluctuating electric fields to positrons in a toroidal dipole field geometry and investigated their effects as a tool to realize radial compression of single particle motions. Experimental setup, single particle orbit analysis, and initial results on the compression experiments are reported in the following sections.

\section{EXPERIMENTAL SETUP AND APPLICATION OF FLUCTUATING FIELDS}

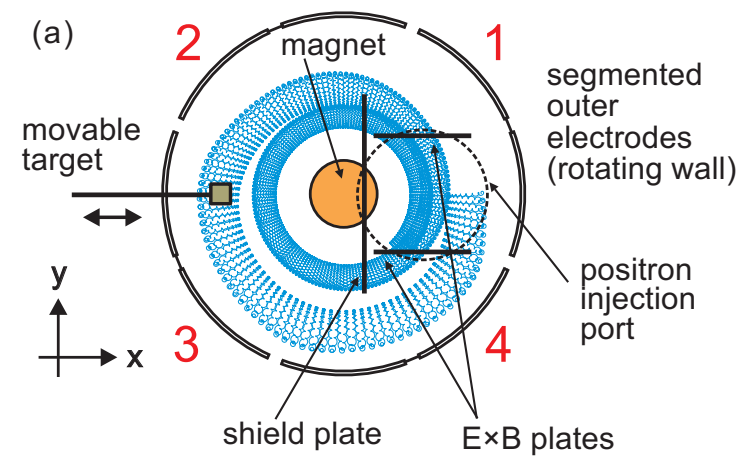

(b)

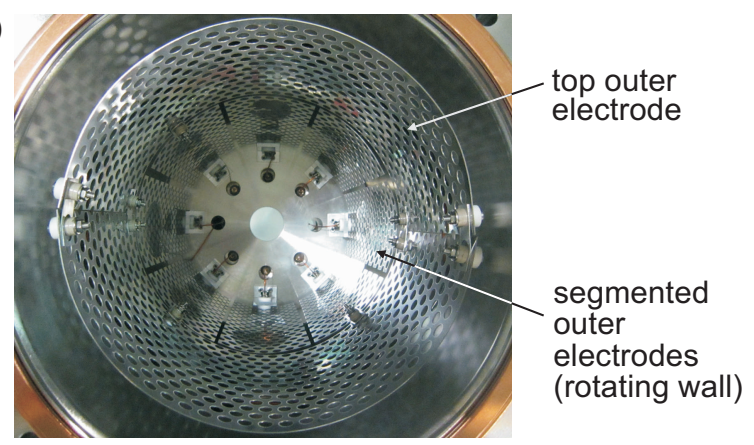

FIGURE 1. (a) Schematic top view and (b) picture of the prototype dipole field trap. Thin lines in (a) show a typical positron orbit in the fluctuating electric fields and magnetic field generated by a permanent magnet and guiding field coils.

The experiments were performed in a prototype dipole field trap [15], shown in Figure 1, at the open beam port of the NEPOMUC facility [8]. The positron beam was transported into the trap with a $5 \mathrm{mT}$ guiding field through an injection port located at the top of the chamber. At the upstream side of the injection port (not shown in the figure), there was a cylindrical deflector electrode used for the fast switching of the beam. In this study, we used $5 \mathrm{eV}$ remoderated positron beam with a typical flux of $2 \times 10^{7} / \mathrm{s}$ [14]. The dipole magnetic field was generated by a neodymium magnet with a surface field strength of $0.6 \mathrm{~T}$ in this prototype experiment, which was mechanically supported by an electrically insulated stainless steel rod. The magnet was located inside a copper case that can be electrically biased against the chamber wall. This was used to control electric fields in the trapping region to realize good confinement configuration for positrons by minimizing the loss cone. Because of the large difference of magnetic field strength, most of field lines in the guiding tube intersected the top of the magnet. In order to inject positrons into the dipole field configuration, local electric fields were applied by a pair of $\mathbf{E} \times \mathbf{B}$ plates that generated cross field drift motion of positrons toward the confinement region. A shield plate is also installed in order to reduce error fields generated by the $\mathbf{E} \times \mathbf{B}$ plates. Efficient injection was realized by adjusting the $\mathbf{E} \times \mathbf{B}$ plate voltages $V_{\mathbf{E} \times \mathbf{B}}$, bias 
voltages of the magnet and outer electrodes, as well as steering coil currents. The status of the injection and trapping experiments at the prototype dipole field trap is presented in [23].
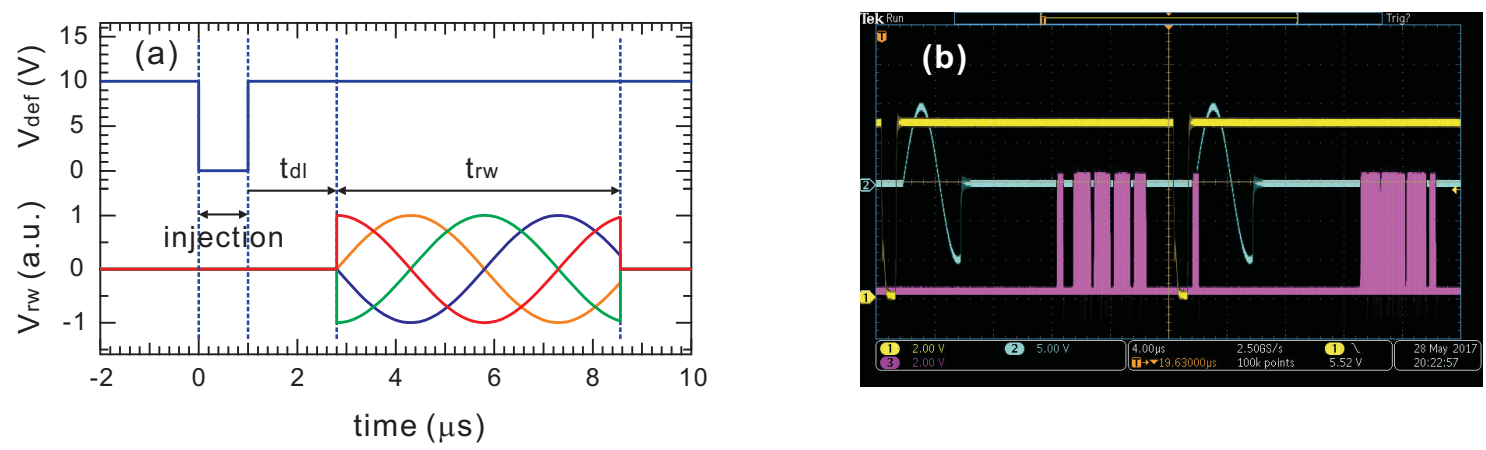

FIGURE 2. (a) A cycle of positron injection and voltage application on each of the outer electrodes. (b) Initial two cycles of the waveforms of (1, yellow) deflector voltage $V_{\text {def }},(2$, cyan) one of the four outer electrode voltages, and (3, purple) $\gamma$-ray signals detected at the target probe in repeated measurement cycles (see more detail in the text).

After injection, positrons make a toroidal precession motion in the dipole field due to the grad-B and curvature drifts, as well as the gyro and bounce motions along magnetic field lines. Typical frequencies of these motions for a $5 \mathrm{eV}$ positron in the present experiment are $f_{\text {gyro }}=100 \mathrm{MHz}, f_{\text {bounce }}=10 \mathrm{MHz}$, and $f_{\text {circ }}=100 \mathrm{kHz}$, respectively. The toroidal precession motion when $E_{\mathrm{r}}=0 \mathrm{~V} / \mathrm{m}$ was in the clockwise direction in the top view of the experiment, as shown in Figure 1 (a). In this trapping phase, fluctuating electric fields were applied to positrons. For this purpose, the outer electrodes were vertically divided into top non-segmented and bottom segmented parts. The bottom insulated electrodes were used as four-fold segments, as shown in the figure. Sinusoidal voltages with a phase difference of $90^{\circ}$,

$$
V_{i}(t)=A_{\mathrm{rw}} \sin \left(2 \pi f_{\mathrm{rw}} t-\theta_{i}\right),
$$

where $\theta_{i}=\pi i / 2$ and $i=1-4$, were applied to the four isolated segments. They generated toroidally rotating electric fields with a toroidal mode number of $n=1$ in the positron trapping region. A typical orbit of a positron under the effects of these electric fields is plotted in Figure 1 (a). The circulation motion of a positron is significantly affected by the electric fields in the frequency range of $f_{\text {circ }}$, resulting in the slow oscillation of the radial position.

In the present experiments, we used pulsed positron beam assuming an application toward future experiments with injection from a positron accumulator. The experimental procedures were as follows (Figure 2 (a)). A pulse of positron beam with a $1 \mu$ s duration, controlled by the electrostatic deflector with an IGBT (insulated gate bipolar transistor) switch, was injected into the trap. After the beam pulse reached the trapping region, the fluctuating fields were applied with various parameters of frequency, amplitude, initial phases, and gating time. Then the spatial profiles of positrons after a $180^{\circ}$ toroidal circulation were measured with a movable target probe located at the opposite side of the injection port. We detected the annihilation $511 \mathrm{keV} \gamma$-ray signals of positrons with two scintillation detectors (BGO crystals). One detector viewed the movable target probe, while the other one viewed the edge of the permanent magnet [15]. Figure 2 (b) shows typical waveforms of the deflector voltage, one of the outer electrode voltages, and $511 \mathrm{keV} \gamma$-ray signals observed at the target probe. When the pulsed beam reached the trapping region of the dipole field, the phase-shifted sinusoidal waves were applied on the outer electrodes for orbit shaping. Then after $180^{\circ}$ toroidal rotation in the trap, positrons hit the target probe. Observations of the $\gamma$-ray signals at the target probe were consistent with these pictures, as shown in the figure. On the actual measurements, the above cycle was repeated in order to improve the data quality. As described in the previous sections, injected positrons toroidally circulate with a time scale in the order of $10 \mu \mathrm{s}$ in the present experiment. In order to realize and observe the radial transport effects before positrons are lost by hitting the target probe, we applied the gated fluctuating fields with frequency of at least more than tens of $\mathrm{kHz}$.

Here it is noted that the fluctuating electric fields affect the orbits of positrons during the injection phase into the trapping region, as well as the radial transport properties of positrons after injection. Experimentally, it is essential to minimize the former effects and to use beam with same injection conditions, in order to precisely investigate the effects of electric fields on positrons trapped in the dipole field. With the above procedures using the pulsed beam and gated fluctuating electric fields, we can investigate their effects on the positron orbit trapped in the dipole field by eliminating their effects on the beam injection conditions. 


\section{POSITRON ORBITS IN A DIPOLE FIELD WITH FLUCTUATING ELECTRIC FIELDS}
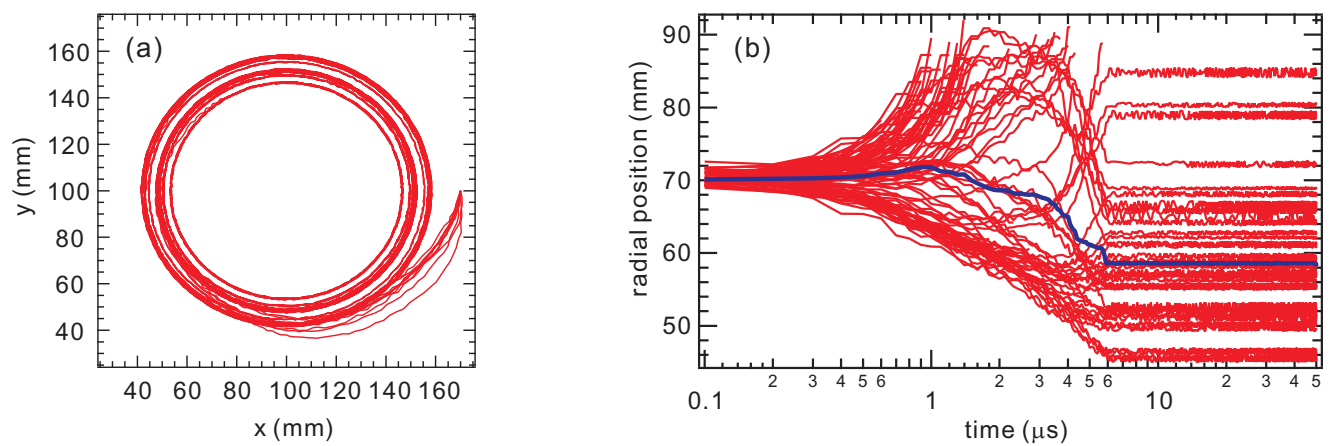

FIGURE 3. (a) Typical orbits (intersection on the $z=0$ plane) that undergo inward drift motions when the fluctuating electric fields of $f_{r w}=150 \mathrm{kHz}$ and $A_{\mathrm{rw}}=7 \mathrm{~V}$ were applied from $t=0$ to $6 \mu \mathrm{s}$. Ten different orbits are superposed. (b) Temporal evolution of the radial positions of positrons when they intersect the $z=0$ plane. The bold line shows the averaged value.
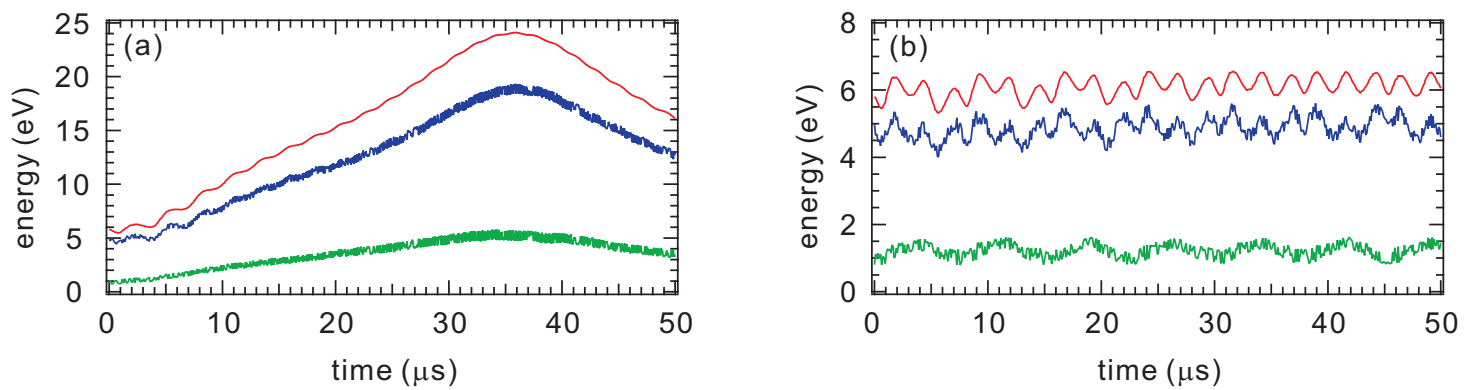

FIGURE 4. Variation of positron energies when crossing the equator plane for fluctuating electric fields with $A_{\mathrm{rw}}=4 \mathrm{~V}$ and frequencies of $f_{\mathrm{rw}}=$ (a) $110 \mathrm{kHz}$, very close to the toroidal circulation frequency of positrons, and (b) $200 \mathrm{kHz}$.

Combination of $E_{\theta}$ and the dipole magnetic field makes cross field drift motion of positrons, resulting in the radial oscillation of the orbit. We can generate such $E_{\theta}$ by applying alternating voltages on toroidally segmented electrodes. Figure 3 shows the examples of positron orbits under the influence of the fluctuating electric fields. The fields were applied in the clockwise $(\mathrm{CW})$ direction, the same direction as the drift circulation motion of positrons, from $t=0$ to $6 \mu \mathrm{s}$. Positrons of $5 \mathrm{eV}$ were injected from $r=70 \mathrm{~mm}$ on the equator plane of the trap with a energy spread of $0.8 \mathrm{eV}$. Because the radial position of positrons undergo slow oscillation, application of gated fields can compress the positron orbits, as shown in the figure. In order to realize efficient compression before dispersion in the initial trapping phase, this oscillation frequency, as well as its amplitude, is an important control parameter.

Application of the fluctuating electric fields can accelerate and heat positrons, which is apparently an undesirable effect for plasma formation. Figure 4 shows the temporal evolution of positron kinetic energies with initial values of $T_{\|}=5.0 \mathrm{eV}$ and $T_{\perp}=0.8 \mathrm{eV}$, when the particle intersected the equator of the experiment. Compared with these initial values, temporal variations of both $T_{\|}$and $T_{\perp}$ are rather moderate except for when the field frequency $f_{\mathrm{rw}}$ is very close to the toroidal circulation frequency $f_{\text {circ }}$ of positrons. It is therefore expected that the heating effects of the fluctuating fields are not a serious problem for the present experimental conditions.

In order to realize efficient compression of the injected positron beam, the effects of finite beam volume in the phase space must be considered. If the motions of particles in the beam are dispersive, rather than the beam is modulated collectively, we cannot use the fluctuating fields as a tool for beam manipulation. In the present experimental conditions, where typically the fluctuating fields in the frequency range of $f_{\text {circ }}$ are applied to $1 \mu$ s pulse of positrons for $5 \mu \mathrm{s}$, we found it is possible to realize macroscopic radial transport before the dispersion effects significantly randomize the positron orbits. Further studies on this point including the spatial and velocity spreads of the beam will be presented elsewhere. 


\section{INITIAL EXPERIMENTAL RESULTS}
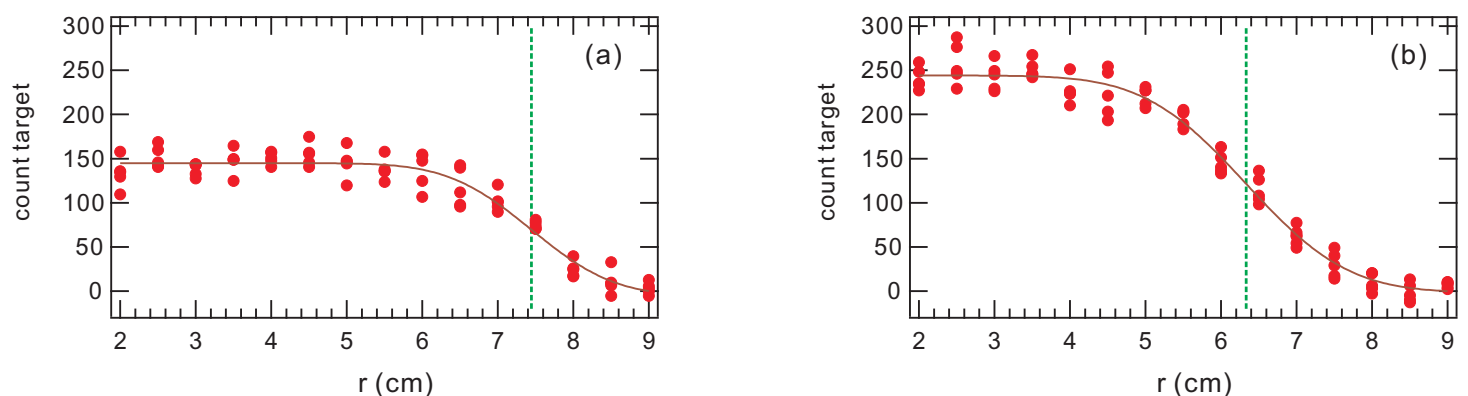

FIGURE 5. Counts of $\gamma$-rays observed at the target probe for various radial target probe position $R_{\text {target }}$, (a) without and (b) with fluctuating fields in CW direction. Dashed lines show the position of the beam center, $r_{\text {beam }}$. The magnet case surface and the outer electrode were located at $r=1.7$ and $9.0 \mathrm{~cm}$.

We conducted positron injection and compression experiments using fluctuating electric fields at NEPOMUC with the following conditions. Steering coil currents $I_{\mathrm{r}}=-8.0 \mathrm{~A}$ and $I_{\theta}=+4.5 \mathrm{~A}$, DC bias voltage of the outer electrodes $V_{r w D C}=0.0 \mathrm{~V}$, top outer electrode voltage $V_{\text {top }}=+16.0 \mathrm{~V}$, magnet bias voltage $V_{\text {mag }}=+5.0 \mathrm{~V}$, and $\mathbf{E} \times \mathbf{B}$ bias plate voltage $V_{\mathbf{E} \times \mathbf{B}}=190.0 \mathrm{~V}$. This is the so-called "injection condition 3" whose typical injection efficiency into the dipole field region was $55 \%$. Positrons were injected as $1 \mu$ s pulses with $20 \mu$ s cycles (5\% duty). The injected positrons were dumped on the target probe as shown in Figure 1 . Annihilation $511 \mathrm{keV} \gamma$-rays were measured with two BGO detectors viewing the target probe (detector 1) and the magnet case (detector 2), for various radial position $R_{\text {target }}$ of the target probe. Here the magnet case and the outer electrodes were located at $R_{\text {target }}=1.7$ and $9.0 \mathrm{~cm}$. We subtract the count levels when $R_{\text {target }}=9.0 \mathrm{~cm}$ as background signals other than those from the target or magnet, including noise signals and $511 \mathrm{keV}$ signals from outer electrodes inside the viewing angle of the detectors. This can be justified because the area of the outer electrode in the viewing angle is very small compared to the entire area.

Figure 5 shows the demonstration of the orbit compression effects. In Figure 5 (b), after positron injection was terminated at $t=0 \mathrm{~s}$, electric fields of $f_{\mathrm{rw}}=200 \mathrm{kHz}$ and $V_{\mathrm{rw}}=10 \mathrm{~V}$ were applied in the CW direction from 0.54 to $3.54 \mu \mathrm{s}$. The graphs show counts from the target probe for various target probe positions. For the $5 \mathrm{eV}$ positron beam, when the target probe was located at $r=R_{\text {target }}$, almost entire positrons located at $R_{\text {target }}<r$ on the equator plane are absorbed by the target probe or its supporting structures on the initial toroidal circulation. The target probe measurements therefore give radial integration of the annihilation counts from the target, as shown in the figure. To find the center position $r_{\text {beam }}$ and spatial spread $\Delta_{\mathrm{r}}$ of the beam in the radial direction, the data were fitted with the error function. When the fluctuating fields were not applied in (a) of the figure, the beam was located at $r_{\text {beam }}=$ $7.4 \mathrm{~cm}$. Radial compression was realized by applying the fields in the $\mathrm{CW}$ direction, as shown in Figure 5 (b), where $r_{\text {beam }}=6.3 \mathrm{~cm}$. In addition to the inward shift of $r_{\text {beam }}$, application of fluctuating fields resulted in $67 \%$ increase of the saturated count $N_{\text {sat }}$, indicating the improved injection efficiency. This is realized through the suppression of positrons loss while traveling from the injection port to the target probe side, by the inward shift of the orbits.

\section{SUMMARY AND FUTURE WORKS}

We reported the first experimental results on the application of fluctuating electric fields to the positron beam injected into the dipole magnetic field configuration. We demonstrated clear modification of the spatial profile of positron beam by applying the fluctuating fields, namely, the inward shift toward the strong field region and enhancement of the injection efficiency. Numerical analysis on the single particle orbits were consistent with the initial experimental results. These results suggest that combination of the $\mathbf{E} \times \mathbf{B}$ drift method and application of the fluctuating fields at the initial injection phase is a useful scheme to realize efficient injection and manipulation of positrons in the dipole field configuration.

In the experiments described here, we focused on simple single particle behavior of positrons under the influence of the fluctuating fields. Here interactions between particles and their collective behaviors were beyond the scope of the present study. Also, effects on the trapping properties caused by the beam manipulation were not investigated in 
the initial experiments. These topics should be investigated in the future. The future research topics also include the trapping time after compression, although this is not straightforward, because the application of fluctuating apparently enhanced the positron loss toward the magnet. Assuming the use of an accumulator in future experiments, studies on the dependence on the pulse length are also important. Another interesting topic is the self-organization processes of charged particles in the dipole field, which can be realized by fluctuating random electric fields as a driving force. Because this is basically a diffusion process realized through the breakdown of $P_{\theta}$, its time scale can be much longer than the toroidal circulation period of the particles. Therefore it is difficult to observe such relaxation processes in the injection and dump experiments conducted in this study. One possible experiment is to estimate the temporal evolution of the spatial profile of long-lived component of positrons by the trapping experiment with and without applying the random voltages on the outer electrodes. Control of the viewing area of the $\gamma$-ray detectors and the use of the target probe as a limiter for the trapping region are also useful to obtain the spatial information of positrons in longer time scales. We will continue the experiments with positrons in the dipole field configuration according to these considerations.

\section{ACKNOWLEDGMENTS}

This work was supported by European Research Council (ERC-2016-ADG No.741322), Germany Research Society (DFG), Max Planck Institute for Plasma Physics, the NIFS Collaboration Research Program, Japan Society for the Promotion of Science (JSPS KAKENHI No. 25707043 and 16KK0094), and the UCSD Foundation.

\section{REFERENCES}

[1] V. Tsytovich and C. B. Wharton, Comments Plasma Phys. Control. Fusion 4, p. 91 (1978).

[2] R. G. Greaves and C. M. Surko, Phys. Rev. Lett. 75, p. 3846 (1995).

[3] W. Oohara and R. Hatakeyama, Phys. Rev. Lett. 91, p. 205005 (2003).

[4] H. Higaki, C. Kaga, F. Fukushima, H. Okamoto, Y. Nagata, Y. Kanai, and Y. Yamazaki, New J. Phys. 19, p. 023016 (2017).

[5] G. Sarri, K. Poder, J. M. Cole, W. Schumaker, A. Di Piazza et al., Nature Comm. 6, p. 6747 (2015).

[6] T. Sunn Pedersen, J. R. Danielson, C. Hugenschmidt, G. Marx, X. Sarasola, F. Schauer, L. Schweikhard, C. M. Surko, and E. Winkler, New J. Physics 14, p. 035010 (2012).

[7] E. V. Stenson, J. Horn-Stanja, M. R. Stoneking, and T. Sunn Pedersen, J. Plasma Phys. 83, p. 595830106 (2017).

[8] C. Hugenschmidt, C. Piochacz, M. Reiner, and K. Schreckenbach, New J. Physics 14, p. 055027 (2012).

[9] Z. Yoshida, Y. Ogawa, J. Morikawa, H. Himura, S. Kondoh, C. Nakashima, S. Kakuno, M. Iqbal, F. Volponi, N. Shibayama, and S. Tahara, AIP Conf. Proc. 498, p. 397 (1999).

[10] Z. Yoshida, H. Saitoh, J. Morikawa, Y. Yano, S. Watanabe, and Y. Ogawa, Phys. Rev. Lett. 104, p. 235004 (2010).

[11] M. Nishiura, Z. Yoshida, H. Saitoh, Y. Yano, Y.Kawazura, T. Nogami, M. Yamasaki, T. Mushiake, and A. Kashyap, Nucl. Fusion 55, p. 053019 (2015).

[12] A. C. Boxer, R. Bergmann, J. L. Ellsworth, D. T. Garnier, J. Kesner, M. E. Mauel, and P. Woskov, Nature Phys. 6, p. 207 (2010).

[13] J. R. Danielson, D. H. E. Dubin, R. G. Greaves, and C. M. Surko, Rev. Mod. Phys. 87, p. 247 (2015).

[14] J. Stanja, U. Hergenhahn, H. Niemann, N. Paschkowski, T. Sunn Pedersen, H. Saitoh, E. V. Stenson, M. R. Stoneking, C. Hugenschmidt, and C. Piochcz, Nucl. Instr. Meth. Phys. Res. A 827, p. 52 (2016).

[15] H. Saitoh, J. Stanja, E. V. Stenson, U. Hergenhahn, H. Niemann, T. Sunn Pedersen, M. R. Stoneking, C. Piochacz, and C Hugenschmidt, New J. Phys. 17, p. 103038 (2015).

[16] A. Hasegawa, Comments Plasma Phys. Control. Fusion 11, p. 147 (1987).

[17] Z. Yoshida, H. Saitoh, Y. Yano, H. Mikami, N. Kasaoka, W. Sakamoto, J. Morikawa, M. Furukawa, and S. M. Mahajan, Plasma Phys. Control. Fusion 55, p. 014018 (2013).

[18] Y. Kawazura, Z. Yoshida, M. Nishiura, H. Saitoh, Y. Yano, T. Nogami, N. Sato, M. Yamasaki, A. Kashyap, and T. Mushiake, Phys. Plasmas 22, p. 112503 (2015).

[19] M. Schulz and L. J. Lanzerotti, Particle Diffusion in the Radiation Belts (Springer, New York, 1974).

[20] X.-P. Huang, F. Anderegg, E. M. Hollmann, C. F. Driscoll, and T. M. O'Neil, Phys. Rev. Lett. 78, p. 875 (1997).

[21] J. R. Danielson and C. M. Surko, Phys. Rev. Lett. 94, p. 035001 (2005).

[22] D. B. Cassidy, R. G. Greaves, V. E. Meligne, and A. P. Mills, Jr., Appl. Phys. Lett. 96, p. 101502 (2010).

[23] U. Hergenhahn, J. Horn-Stanja et al., AIP Conference Proc. (this volume) (2017). 